\title{
NOTA
}

\section{EFEITO DA SOLUÇÃO DE SEPARAÇÃO DENSIMÉTRICA NA QUANTIDADE E QUALIDADE DA MATÉRIA ORGÂNICA LEVE E NA QUANTIFICAÇÃO DO CARBONO ORGÂNICO DA FRAÇÃO PESADA ${ }^{(1)}$}

\author{
Michelle Sales Moreira Demolinari(2), Ivo Ribeiro Silva ${ }^{(3)}$, Augusto \\ Miguel Nascimento Lima ${ }^{(4)}$, Leonardus Vergutz ${ }^{(4)} \&$ Eduardo de Sá \\ Mendonça $^{(5)}$
}

\section{RESUMO}

\begin{abstract}
A matéria orgânica leve tem se mostrado um indicador precoce das mudanças na matéria orgânica do solo (MOS) acarretadas por diferentes usos e sistemas de manejo. Várias soluções são empregadas na sua separação, mas pouco se sabe sobre o efeito delas na quantidade e qualidade da fração extraída, tampouco se conhecem as possíveis interferências que a solução de separação pode ocasionar na posterior quantificação do carbono (C) orgânico. Assim, foram selecionados 20 solos com diferentes teores de argila e que se encontravam sob diferentes usos, onde foram coletadas amostras, em três repetições, na profundidade de 0-20 cm. Depois de secas ao ar, destorroadas, passadas em peneira com malha de $2 \mathrm{~mm}$ e homogeneizadas, retiraram-se subamostras para determinação do teor de argila e para realização do fracionamento densimétrico da MOS. Do fracionamento densimétrico com água ou $\mathrm{NaI}\left(1,8 \mathrm{~kg} \mathrm{~L}^{-1}\right)$, foram obtidas a fração leve (FL) e a fração pesada (FP) da MOS. O teor de C da FL foi determinado por combustão via seca, em analisador elementar (CHN), e o teor de C da FP foi determinado por oxidação via úmida com aquecimento externo, segundo Yeomans \& Bremner (1988)
\end{abstract}

\footnotetext{
(1) Recebido para publicação em janeiro de 2007 e aprovado em novembro de 2007.

${ }^{(2)}$ Mestranda em Solos e Nutrição de Plantas, Universidade Federal de Viçosa - UFV. Av. PH Rolfs s/n, CEP 36570-000 Viçosa (MG). Bolsista da CAPES.

(3) Professor Adjunto do Departamento de Solos, UFV. E-mail: ivosilva@ufv.br

(4) Doutorando do Departamento de Solos e Nutrição de Plantas, UFV. Bolsista CNPq.

(5) Professor Associado do Departamento de Solos, UFV. E-mail: esm@ufv.br
} 
(Y \& B), e em analisador elementar (CHN). Adicionalmente, foi determinado o teor equivalente em $C$ orgânico por via úmida em soluções-padrão com $\mathrm{NaI}$ em diferentes concentrações. Foi observado que a quantidade da FL extraída com NaI foi, em média, 4,86 vezes maior em relação à extraída com água destilada. A relação $\mathrm{C}: \mathrm{N}$ da fração extraída com $\mathrm{NaI}$ foi, em média, 1,85 vez superior à apresentada pela extração com água destilada, mas com grande variação entre solos. Com relação à $\mathrm{FP}$ da MOS, o teor de $\mathrm{C}$ determinado por Y \& $\mathrm{B}$ foi similar quando se procedeu à separação densimétrica com água, mas foi cerca de $1,5 \mathrm{vez}$ superior ao determinado pelo CHN quando esta fração foi separada com NaI, indicando que houve superestimação do teor de $\mathrm{C}$ orgânico devido à interferência do $\mathrm{NaI}$. Esse fato foi confirmado pelo aumento linear no consumo de $\mathrm{Cr}_{2} \mathrm{O}_{7}^{-2}$ quando da presença de concentrações crescentes de $\mathrm{NaI}$ em soluções isentas de C. Dessa forma, a extração com NaI recupera maior proporção da FL, mas que difere em qualidade daquela extraída com água. Além disso, algum possível resíduo de NaI usado na separação pode interferir na quantificação do $\mathrm{C}$ orgânico quando este for determinado por dicromatometria. Resultados de estudo adicional sugerem que cada mol de $\mathrm{I}^{-}$consome aproximadamente $1 \mathrm{~mol} \mathrm{de} \mathrm{Cr}_{2} \mathrm{O}_{7}^{-2}$.

Termos de indexação: matéria orgânica, fracionamento densimétrico, iodeto de sódio.

\section{SUMMARY: THE EFFECT OF DENSITY FRACTIONATION SOLUTIONS ON THE AMOUNT AND QUALITY OF LIGHT-FRACTION ORGANIC MATTER AND THE CONTENT OF HEAVY-FRACTIONORGANIC $C A R B O N$}

The light organic matter fraction is known as an early indicator of changes in soil organic matter (SOM) as a result of different soil use and management. Several solutions are used in its separation, but little is known about the effect of these solutions on the quantity and quality of the extracted fraction, as well as the possible interferences of the separation solution on the quantification of organic carbon $(C)$ content. Twenty soils with variable clay contents and under different uses were selected. Soil samples were collected in the 0-20 cm layer, with three replications. After air-drying, they were ground manually, passed through a $2 \mathrm{~mm}$ sieve and homogenized; sub-samples were taken for clay content determination and densimetric SOM fractionation. Based on the fractionation scheme using water or $\mathrm{NaI}\left(1.8 \mathrm{~kg} \mathrm{L^{-1 }}\right)$ the SOM light fraction (LF) and the heavy fraction (HF) were obtained. The $C$ concentration of $L F$ was determined by dry combustion in an elemental analyzer $(\mathrm{CHN})$ and the $C$ content of $\mathrm{HF}$ by wet oxidation, according to Yeomans \& Bremner (1988) (Y \& B) and by dry combustion. Additionally, we determined the equivalent organic $C$ concentration by wet oxidation of standard solutions containing increasing $\mathrm{NaI}$ concentration. It was found that the amount of NaI-extracted LF averaged across soils was 4.86 times greater than when extracted with distilled water. The $C: N$ ratio of this fraction was, on average, 1.85 times greater than that extracted with water, and the variation among soils was high. With regard to $H F$, the C concentration determined by wet oxidation was similar to that separated with water, but 1.5 times larger than when determined by dry combustion if separated with $\mathrm{NaI}$. These results indicate that the $C$ content was overestimated, possibly due to the interference of NaI. This finding was confirmed by the linear increase in the consumption of $\mathrm{Cr}_{2} \mathrm{O}_{7}{ }^{-2}$ during organic $C$ determination in the presence of increasing NaI concentration of organic- $C$ free solutions. Therefore, the NaI extraction of $L F$ recovers a larger amount of $L F$, which differs however in quality from that extracted with water. Additionally, any potential NaI remaining after the separation, especially in the $H F$, may interfere with the organic $C$ quantification if wet oxidation with dichromate as oxidant agent is the chosen method. Results of a complementary study indicate that each mol of I consumes approximately $1 \mathrm{~mol}$ of $\mathrm{Cr}_{2} \mathrm{O}_{7}{ }^{-2}$.

Index terms: organic matter, densimetric fractionation, sodium iodide. 


\section{INTRODUÇÃO}

A matéria orgânica do solo (MOS) é composta por várias frações que diferem quanto às características físicas e químicas e ao tempo de ciclagem (Stevenson, 1994; Liao et al., 2006), fato que pode contribuir para que alterações em determinadas frações da MOS por diferentes usos e manejos do solo possam levar anos para serem detectadas.

Fisicamente, a MOS tem sido operacionalmente dividida em fração leve (FL) e fração pesada (FP) (Sohi et al., 2001). A FL é constituída por restos vegetais macroscópicos parcialmente decompostos, localizada inter e intra-agregados do solo, enquanto a FP é aquela que se encontra no solo protegida física e, ou, quimicamente, correspondendo à matéria orgânica associada às frações minerais do solo (areia, silte e argila), estando, na sua maioria, em estádio mais avançado de decomposição.

A FLé uma fonte de $\mathrm{C}$ e nutrientes mais disponível aos organismos do solo, tendo tempo de ciclagem mais reduzido (Swanston et al., 2005). A decomposição da FL da MOS é determinada pelos fatores relacionados a clima, práticas de manejo e características químicas intrínsecas ao próprio material orgânico (Christensen, 2001). A FL apresenta características semelhantes às do material orgânico que lhe deu origem, enquanto a FP apresenta maior grau de transformação, sendo parte de origem microbiana (Poirier et al., 2005). Apesar de a maior proporção do C orgânico total (COT) do solo estar associada à FP (Roscoe \& Buurman, 2003; Lima, 2004; Mando, et al., 2005; Poirier et al., 2005), esta fração apresenta mudanças mais lentas e graduais, fazendo com que a FL seja considerada como indicador mais sensível à mudança na qualidade $\mathrm{e}$ uso do solo (Garten Jr. et al., 1999; Freixo et al., 2002a,b; Wu et al., 2004).

O fracionamento densimétrico da MOS constitui uma importante ferramenta para verificar possíveis alterações quantitativas e qualitativas na MOS pela mudança de uso no curto prazo (Sohi et al., 2001) e pode auxiliar na escolha de práticas de manejo que maximizem a eficiência de uso de água e nutrientes (Marriott \& Wander, 2006). O fracionamento densimétrico da MOS tem como princípio a separação de frações da MOS (FL e FP) com base na densidade dos componentes do solo em meio líquido de densidade conhecida (Christensen, 2001; Six et al., 1998, 2001, 2002; Liao et al., 2006). Diversos trabalhos têm usado o fracionamento densimétrico da MOS; alguns deles utilizaram solução de iodeto de $\mathrm{Na}\left(\mathrm{NaI} 1,8 \mathrm{~g} \mathrm{~cm}^{-3}\right)$ (Sollins et al.,1984; Sohi et al., 2001; Lima, 2004; Poirier et al., 2005), enquanto outros empregaram água destilada (Kandeler et al., 1999; Quédraogo et al., 2005; Liao et al., 2006). Adicionalmente, alguns trabalhos têm utilizado outras soluções, como politungstato de $\mathrm{Na}\left(1,6-1,7 \mathrm{~g} \mathrm{~cm}^{-3}\right)$ (Swanston et al., 2005; Marriott \& Wander, 2006), o que muitas vezes tem gerado dificuldade quando da comparação dos resultados entre trabalhos.
Além de o NaI ser de dispendiosa aquisição, vários problemas de insalubridade são observados pela inalação, como enxaqueca, vertigem, fraqueza e edema pulmonar; sua ingestão causa náuseas, vômitos e diarréia; e o contato com a pele provoca vermelhidões e até mesmo erupções cutâneas de pele (MSDS Solutions, 2007).

O uso de soluções com densidade e composição diferentes pode resultar na separação de FL distinta, tanto quantitativa, quanto qualitativamente (Sohi et al., 2001). Adicionalmente, algum resíduo da solução utilizada na separação densimétrica da MOS pode interferir na quantificação dos teores de C orgânico, especialmente da FP, que possui maior superfície específica e interação com a fase mineral do solo.

Dessa forma, este trabalho teve como objetivos: comparar a quantidade e a qualidade da FL e FP obtidas por meio do fracionamento densimétrico da MOS com NaI e água destilada; e avaliar as possíveis interferências do NaI usado na separação de FL e FP na quantificação do $\mathrm{C}$ orgânico via úmida por dicromatometria.

\section{MATERIAL E MÉTODOS}

Foram selecionados 20 solos, visando cobrir uma ampla faixa de teores de argila (7 a $68 \%$ ), para os quais foi realizado o fracionamento densimétrico da MOS, utilizando solução de $\mathrm{NaI}$ ou água destilada. $\mathrm{Na}$ época da coleta (primeiro semestre de 2002), eles se encontravam sob mata nativa, pastagem plantada ou culturas anuais. Detalhes adicionais sobre as características químicas desses solos podem ser consultados no trabalho de Milagres (2003). Os tratamentos consistiram de um fatorial $2 \times 2$ (duas soluções de separação e dois métodos de quantificação do COT), num delineamento inteiramente casualizado, com três repetições.

As amostras de cada solo foram coletadas na profundidade de $0-20 \mathrm{~cm}$, em três repetições. Em seguida, elas foram secas ao ar, destorroadas, passadas em peneira com abertura de malha de $2 \mathrm{~mm}$ e homogeneizadas, obtendo-se a terra fina seca ao ar (TFSA). A partir dessas amostras de TFSA, retiraram-se subamostras para determinação do teor de argila pelo método da pipeta (Embrapa, 1997) e COT por meio de combustão via úmida, segundo método proposto por Walkley \& Black (Defelipo \& Ribeiro, 1997).

Nas amostras de solo foi realizado o fracionamento densimétrico da MOS, utilizando $\mathrm{NaI}\left(1,8 \mathrm{~kg} \mathrm{~L}^{-1}\right)$ ou água destilada. Resumidamente, o método consistiu em agitar $15 \mathrm{~g}$ de TFSA com $30 \mathrm{~mL}$ de NaI $\left(1,8 \mathrm{~kg} \mathrm{~L}^{-1}\right.$, o que corresponde a aproximadamente $12 \mathrm{~mol} \mathrm{~L}^{-1}$ ) ou água destilada, em tubos de centrífuga de $50 \mathrm{~mL}$ 
durante $16 \mathrm{~h}$ em agitador vertical, não se fazendo uso de dispersante químico. Em seguida, centrifugaram-se as amostras a $630 \mathrm{~g}$ por $15 \mathrm{~min}$, e o sobrenadante foi passado em peneira de 100 mesh $(0,149 \mathrm{~mm})$ para coletar a fração leve (FL) da matéria orgânica. Como o procedimento adotado envolveu o rompimento dos agregados por agitação contínua antes da separação, a fração leve isolada neste estudo é constituída pelo compartimento inter e intra-agregados (Sohi et al., 2001). A matéria orgânica associada aos componentes minerais do solo e que sedimentou no fundo do tubo de centrífuga foi considerada a fração pesada (FP). Após a separação, a FL foi seca em estufa a $72^{\circ} \mathrm{C}$ por $72 \mathrm{~h}$ e, em seguida, pesada e macerada em almofariz de ágata, para então se realizar a determinação do teor de C por combustão via seca, em analisador elementar (CHN). A FP do fundo do tubo de centrífuga foi lavada duas vezes com $30 \mathrm{~mL}$ de água destilada, a fim de remover o excesso de $\mathrm{NaI}$ adsorvido à fração mineral do solo, e, em seguida, centrifugada, seca em estufa a $72{ }^{\circ} \mathrm{C}$ por $72 \mathrm{~h}$, macerada em almofariz de porcelana e passada em peneira de 100 mesh $(0,149 \mathrm{~mm})$, para determinação do teor de C. Para avaliar se o $\mathrm{NaI}$ usado na solução de separação densimétrica da MOS afetou a posterior quantificação do $\mathrm{C}$ orgânico por dicromatometria, os teores de C orgânico da FP proveniente de amostras separadas com $\mathrm{NaI}$ ou água foram determinados por oxidação via úmida com aquecimento externo $\left(170^{\circ} \mathrm{C}\right.$ durante 30 min em bloco pré-aquecido), proposto por Yeomans \& Bremner (1988), e por combustão via seca em analisador elementar $(\mathrm{CHN})$, considerado método de referência.

Um estudo adicional foi realizado visando avaliar a interferência do $\mathrm{NaI}$ no consumo do agente oxidante (dicromato), o que, por sua vez, resultou em estimativas incorretas dos teores de $\mathrm{C}$ orgânico do solo. Para isso, soluções com concentrações crescentes de $\mathrm{NaI}(0 ; 0,12 ; 0,24 ; 0,35 ; 0,47 ; 0,59 ; 0,71 ; 0,83 ; 0,94$; 1,06; e $1,18 \mathrm{~mol} \mathrm{~L}^{-1}$ ) isentas de $\mathrm{C}$ orgânico foram submetidas à oxidação com solução de dicromato, seguindo o procedimento de determinação de COT (Yeomans \& Bremner, 1988). É importante ressaltar que nenhuma fonte de carbono foi acrescida nas soluções, a fim de quantificar apenas a possível interferência do $\mathrm{NaI}$ na quantificação de carbono. Logo, esperava-se que, se não houvesse interferência, o consumo do agente oxidante fosse nulo.

A massa, os teores de $\mathrm{C}$, as relações elementares da FL e os teores de COT e C da FP foram submetidos à análise de variância e comparados por meio do teste de Tukey $(\alpha=0,05)$, utilizando-se o software SAEG 9.0 (Funarbe, 2005).

Quadro 1. Classe, procedência, características granulométricas e matéria orgânica dos solos

\begin{tabular}{|c|c|c|c|c|c|c|c|}
\hline Solo & Classe & Uso & Procedência & Argila $^{(1)}$ & Silte ${ }^{(1)}$ & $\operatorname{Areia}^{(1)}$ & $\operatorname{COT}^{(2)}$ \\
\hline & & & & & $-\%$ & - & $\mathrm{g} \mathrm{kg}^{-1}$ \\
\hline 1 & Neossolo & Pastagem & Ilhéus - BA & 7 & 2 & 91 & 9,7 \\
\hline 2 & Chernossolo & Pastagem & Itaju do Colônia - BA & 12 & 11 & 77 & 2,3 \\
\hline 3 & Vertissolo & Pastagem & Itaju do Colônia - BA & 14 & 19 & 67 & 10,9 \\
\hline 4 & Planossolo & Pastagem & Itaju do Colônia - BA & 16 & 20 & 64 & 10,2 \\
\hline 5 & Latossolo & Agricultura & São João Evangelista - MG & 17 & 6 & 77 & 5,5 \\
\hline 6 & Cambissolo & Pastagem & Viçosa - MG & 24 & 8 & 68 & 12,1 \\
\hline 7 & Argissolo & Floresta & Mascote - BA & 26 & 32 & 42 & 28,2 \\
\hline 8 & Latossolo & Agricultura & São João Evangelista - MG & 32 & 12 & 56 & 17,9 \\
\hline 9 & Latossolo & Floresta & Senhora de Oliveira - MG & 38 & 20 & 41 & 16,0 \\
\hline 10 & Latossolo & Agricultura & São João Evangelista - MG & 40 & 16 & 44 & 17,2 \\
\hline 11 & Latossolo & Agricultura & Peçanha - MG & 42 & 22 & 36 & 37,0 \\
\hline 12 & Latossolo & Pastagem & São João Evangelista - MG & 45 & 26 & 29 & 26,9 \\
\hline 13 & Latossolo & Floresta & São João Evangelista - MG & 50 & 7 & 43 & 17,5 \\
\hline 14 & Latossolo & Pastagem & São João Evangelista - MG & 52 & 9 & 39 & 18,7 \\
\hline 15 & Latossolo & Floresta & Senhora de Oliveira - MG & 54 & 10 & 36 & 35,8 \\
\hline 16 & Latossolo & Floresta & Senhora de Oliveira - MG & 57 & 9 & 34 & 44,0 \\
\hline 17 & Latossolo & Pastagem & Senhora de Oliveira - MG & 58 & 9 & 33 & 8,6 \\
\hline 18 & Latossolo & Floresta & São João Evangelista - MG & 62 & 0 & 38 & 22,6 \\
\hline 19 & Latossolo & Agricultura & Senhora de Oliveira - MG & 65 & 16 & 19 & 17,9 \\
\hline 20 & Latossolo & Floresta & São João Evangelista - MG & 68 & 3 & 29 & 30,8 \\
\hline
\end{tabular}

(1) Embrapa, 1997; ${ }^{(2)}$ Método Walkley \& Black (Defelipo \& Ribeiro, 1997). Fonte: Milagres (2003). 


\section{RESULTADOS E DISCUSSÃO}

A massa da fração leve da MOS (FL) separada com $\mathrm{NaI}\left(1,8 \mathrm{~kg} \mathrm{~L}^{-1}\right)$ foi, em média, 4,86 vezes maior que a extraída com água (Figura 1 ). Em razão de a densidade do $\mathrm{NaI}\left(1,8 \mathrm{~kg} \mathrm{~L}^{-1}\right)$ ser superior a da água $\left(1,0 \mathrm{~kg} \mathrm{~L}^{-1}\right)$, o poder de extração do NaI é maior. Além disso, o Na, por ser um íon que apresenta elevado raio hidratado, contribui para quebra de agregados por meio da dispersão do solo, facilitando assim a remoção de maior quantidade da FL. Dessa forma, as frações de matéria orgânica que apresentam valor de densidade entre 1,0 e $1,8 \mathrm{~kg} \mathrm{~L}^{-1}$, que ficariam em suspensão quando se utilizou o NaI como solução de separação, sedimentaram quando se utilizou água como solução de separação. Em outros estudos também tem-se observado que líquidos mais densos possuem maior capacidade de extração da FL (Sohi et al., 2001).

Além das diferentes quantidades extraídas da FL, também foram observadas diferenças na qualidade da FL extraída com as duas soluções, refletidas na sua composição elementar. A FL extraída com NaI apresentou relação $\mathrm{C}: \mathrm{N}$, em média, 1,85 vez superior à relação $\mathrm{C}: \mathrm{N}$ apresentada pela mesma fração extraída com água destilada (Quadro 2). A média geral deste estudo pode levar a resultados conflitantes, pois esperase que, em virtude de o material extraído com a água ser de menor densidade, ele seja parecido mais com o material vegetal que lhe deu origem (Poirier et al., 2005; Marriott \& Wander, 2006; Liao et al., 2006), em estádio menos avançado de decomposição (ex.: resíduos vegetais parcialmente decompostos), em comparação com o material extraído com iodeto. Dessa forma, maior relação $\mathrm{C}: \mathrm{N}$ seria esperada para a FL recuperada com água. De fato, em alguns dos solos utilizados, a FL recuperada com água apresentou maior relação C:N, mas isso não foi consistente para

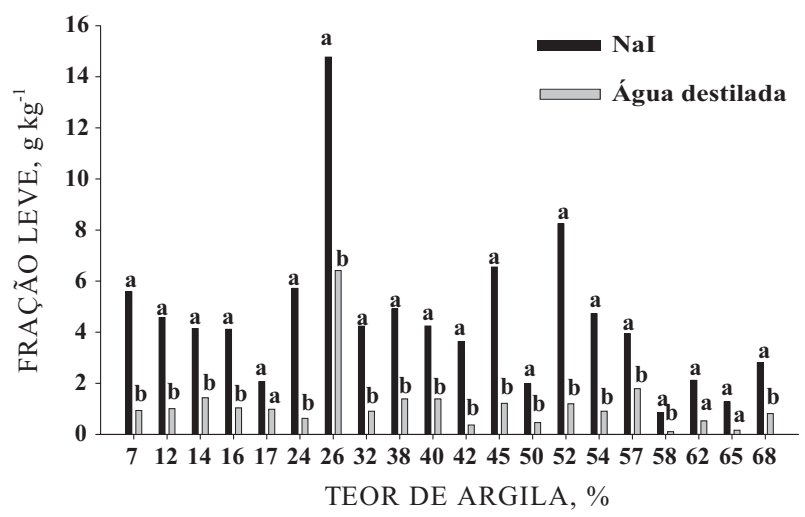

Figura 1. Massa da fração leve (FL) da matéria orgânica do solo ( $\mathrm{g} \mathrm{kg}^{-1}$ de solo) extraída com iodeto de sódio (NaI) e água destilada. Letras iguais para cada solo com o mesmo teor de argila não diferem quanto aos teores de carbono a $5 \%$ pelo teste de Tukey. todos os solos. No entanto, esses resultados podem ser conciliados, pois a extração com $\mathrm{NaI}$ recuperou uma FL com menor teor de C em comparação com aquela extraída com água (Quadro 2), sugerindo que ela, de fato, se encontre em estádio mais avançado de decomposição.

A relação $\mathrm{H}: \mathrm{C}$ tem sido usada como indicador do grau de insaturação de compostos orgânicos (Yonebayashi \& Hattori, 1988; Conte et al., 2003) e está relacionada com sua taxa de mineralização (Martin et al., 1998). Menor relação H:C implica maior grau de insaturação de materiais húmicos (Stevenson, 1994). De maneira geral, a FL extraída com $\mathrm{NaI}$ e com água não diferiu quanto ao valor da relação H:C (1,51 versus 1,55) (Quadro 2). Contudo, para os solos mais arenosos, com teor de argila variando entre 7 e $24 \%$, a relação H:C da FL extraída com $\mathrm{NaI}$ foi menor do que quando extraída com água. Assim, para esses solos, o NaI estaria recuperando uma porção da FL quimicamente distinta, resultando em maior grau de insaturação desta fração.

Nos solos com teor de argila acima desse intervalo não se observou tendência muito clara quanto ao valor da relação H:C. Ficou evidente, no entanto, que praticamente em todos os solos a FL extraída com água possui maior teor de $\mathrm{H}$ que aquela extraída com NaI, novamente indicando que ela se encontra em estádio mais avançado de decomposição e, possivelmente, inclui produtos intermediários da atividade microbiana.

A maior parte da MOS está associada aos componentes minerais do solo (fração pesada, FP). As análises dessa fração indicam que, de maneira geral, o teor de C orgânico determinado pelo método de Yeomans \& Bremner (Y \& B) praticamente não diferiu daquele determinado pelo analisador elementar (CHN) quando esta fração foi separada com água destilada (Figura 2).

No entanto, quando a FP era derivada de amostras de solo que foram fracionadas densimetricamente com solução de $\mathrm{NaI}$, o teor de $\mathrm{C}$ determinado pelo método de Y \& B foi, em média, 1,5 vez superior àquele determinado por combustão a seco em analisador elementar. Solos com maior teor de argila (> $26 \%$ ) podem dificultar mais a lavagem da FP, fazendo com que o excesso de solução de $\mathrm{NaI}$ não seja facilmente retirado, gerando maior interferência na medição do $\mathrm{C}$ por via úmida (Figura 2). No entanto, não se pode descartar um efeito da mineralogia do solo, pois, segundo Wieseman \& Püttmann (2006), além da textura do solo, a sua mineralogia pode exercer forte influência na sorção e estabilização da MOS. Isso mostra que, na determinação do C orgânico por via úmida, utilizando-se um oxidante químico, pode estar ocorrendo reação do NaI residual (I- na forma reduzida) com o dicromato. O método de determinação do $\mathrm{C}$ orgânico por dicromatometria é um dos mais antigos e difundidos, principalmente devido à sua simplicidade e ao requerimento de equipamentos acessíveis para a 
Quadro 2. Teores de carbono, hidrogênio, nitrogênio e relações molares C:N e H:C da fração leve da matéria orgânica com iodeto de sódio (NaI) e com água destilada

\begin{tabular}{|c|c|c|c|c|c|c|c|c|c|c|}
\hline \multirow{2}{*}{ Solo } & \multicolumn{5}{|c|}{ Iodeto de sódio $\left(1,8 \mathrm{~kg} \mathrm{~L}^{-1}\right)$} & \multicolumn{5}{|c|}{ Água destilada } \\
\hline & C & $\mathbf{H}$ & $\mathbf{N}$ & $\mathrm{C}: \mathrm{N}$ & $\mathrm{H}: \mathrm{C}$ & C & $\mathbf{H}$ & $\mathbf{N}$ & $\mathrm{C}: \mathrm{N}$ & $\mathrm{H}: \mathrm{C}$ \\
\hline & \multicolumn{3}{|c|}{$\%$} & & & \multicolumn{3}{|c|}{$-\%$} & & \\
\hline 1 & 19,61 & 1,54 & 0,05 & 392,2 & 0,94 & 32,71 & 4,59 & 0,48 & 68,15 & 1,68 \\
\hline 2 & 11,72 & 0,86 & 0,12 & 97,7 & 0,88 & 33,52 & 4,5 & 0,47 & 71,32 & 1,61 \\
\hline 3 & 23,65 & 2,29 & 0,54 & 43,8 & 1,16 & 27,06 & 4,73 & 0,46 & 58,83 & 2,10 \\
\hline 4 & 17,05 & 1,71 & 0,54 & 31,6 & 1,20 & 34,37 & 4,66 & 0,87 & 39,51 & 1,63 \\
\hline 5 & 22,89 & 2,2 & 0,9 & 25,4 & 1,15 & 23,17 & 4,08 & 0,07 & 331,00 & 2,11 \\
\hline 6 & 17,57 & 1,34 & 0,31 & 56,7 & 0,92 & 30,95 & 4,12 & 0,07 & 442,14 & 1,60 \\
\hline 7 & 21,63 & 2,83 & 1,02 & 21,2 & 1,57 & 37,03 & 4,29 & 1,12 & 33,06 & 1,39 \\
\hline 8 & 18,79 & 2,79 & 0,84 & 22,4 & 1,78 & 32,31 & 4,85 & 0,53 & 60,96 & 1,80 \\
\hline 9 & 18,04 & 2,71 & 0,36 & 50,1 & 1,80 & 30,58 & 4,42 & 0,45 & 67,96 & 1,73 \\
\hline 10 & 21,22 & 2,91 & 0,64 & 33,2 & 1,65 & 34,61 & 4,04 & 0,7 & 49,44 & 1,40 \\
\hline 11 & 25,01 & 2,83 & 0,51 & 49,0 & 1,36 & 37,95 & 4,25 & 1,12 & 33,88 & 1,34 \\
\hline 12 & 20,55 & 3,32 & 0,1 & 205,5 & 1,94 & 35,89 & 4,42 & 0,39 & 92,03 & 1,48 \\
\hline 13 & 23,41 & 3,26 & 0,17 & 137,7 & 1,67 & 27,67 & 3,67 & 0,44 & 62,89 & 1,59 \\
\hline 14 & 15,48 & 2,56 & 0,36 & 43,0 & 1,98 & 33,25 & 4,21 & 0,53 & 62,74 & 1,52 \\
\hline 15 & 17,26 & 2,31 & 0,09 & 191,8 & 1,61 & 38,39 & 4,00 & 1,69 & 22,72 & 1,25 \\
\hline 16 & ND & ND & ND & ND & ND & 35,14 & 3,76 & 0,68 & 51,68 & 1,28 \\
\hline 17 & 11,73 & 2,68 & 0,05 & 234,6 & 2,74 & 28,32 & 3,56 & 0,64 & 44,25 & 1,51 \\
\hline 18 & 36,06 & 3,54 & 0,81 & 44,5 & 1,18 & 25,52 & 2,97 & 0,74 & 34,49 & 1,40 \\
\hline 19 & 23,18 & 3,14 & 0,47 & 49,3 & 1,63 & 30,89 & 3,66 & 0,6 & 51,48 & 1,42 \\
\hline 20 & 20,8 & 2,59 & 0,36 & 57,8 & 1,49 & 40,03 & 3,96 & 0,9 & 44,48 & 1,19 \\
\hline
\end{tabular}

ND: não determinado.

maioria dos laboratórios. Neste método é utilizado o dicromato $\left(\mathrm{Cr}_{2} \mathrm{O}_{7}{ }^{2-}\right)$ como agente oxidante em meio ácido, sem aquecimento externo (Walkley \& Black, 1934), ou com aquecimento externo, conforme proposto por Yeomans \& Bremner (1988). Dessa forma, assumese que a reação de oxidação do $\mathrm{C}$ orgânico seja a demonstrada na equação seguinte:

$$
2 \mathrm{Cr}_{2} \mathrm{O}_{7}^{2-}+3 \mathrm{C}^{\circ}+16 \mathrm{H}^{+} \leftrightarrow 4 \mathrm{Cr}^{3+}+3 \mathrm{CO}_{2}+8 \mathrm{H}_{2} \mathrm{O}
$$

A titulação do dicromato é feita com uma solução de $\mathrm{Fe}$ reduzido $\left(\mathrm{FeSO}_{4} .7 \mathrm{H}_{2} \mathrm{O}\right)$ em meio ácido, empregando-se como indicador difenilamina, conforme a reação descrita na equação 2 :

$$
\mathrm{Cr}_{2} \mathrm{O}_{7}^{2-}+6 \mathrm{Fe}^{2+}+14 \mathrm{H}^{+} \leftrightarrow 2 \mathrm{Cr}^{3+}+6 \mathrm{Fe}^{2+}+7 \mathrm{H}_{2} \mathrm{O}
$$

Uma das suposições do método é de que todo o $\mathrm{C}$ orgânico do solo encontra-se na forma reduzida $\mathrm{C}^{\circ} \mathrm{e}$ que o dicromato consumido durante as reações se deve à oxidação do $\mathrm{C}$ orgânico. Interferências de vários íons facilmente oxidáveis e que podem ser encontrados no solo, como o $\mathrm{Cl}^{-}, \mathrm{Fe}^{2+}$ e $\mathrm{MnO}_{2}$, têm sido reportadas (Walkley, 1947). Mais recentemente, o fracionamento densimétrico da MOS tem ganhado importância, e vários trabalhos têm utilizado soluções mais densas que a água para separar maior proporção da FL da MOS. O NaI é um dos sais freqüentemente usados no preparo dessas soluções (Sohi et al., 2001; Lima, 2004; Poirier et al., 2005). Nesses trabalhos, o teor de C da FL e da FP separada com essas soluções de alta densidade é normalmente determinado por combustão via seca.

No entanto, analisadores elementares são equipamentos relativamente caros e que não se encontram disponíveis na maioria dos laboratórios que desenvolvem estudos com a MOS. Os dados deste estudo mostram que a quantificação do $\mathrm{C}$ orgânico associada a essas frações pesadas utilizando-se o dicromato $\left(\mathrm{Cr}_{2} \mathrm{O}_{7}^{2-}\right)\left(\mathrm{Cr}^{6+}\right)$ pode sofrer influência do residual de NaI, mesmo após lavagem do solo com água em abundância, levando à superestimação do teor de C orgânico. No entanto, não foram encontrados trabalhos na literatura que abordassem a interferência do I' nesse método.

A possibilidade de reação do I' com dicromato foi confirmada em estudo complementar, em que se observou que concentrações crescentes de $\mathrm{NaI}$ em solução isenta de $\mathrm{C}$ orgânico levaram ao incremento 


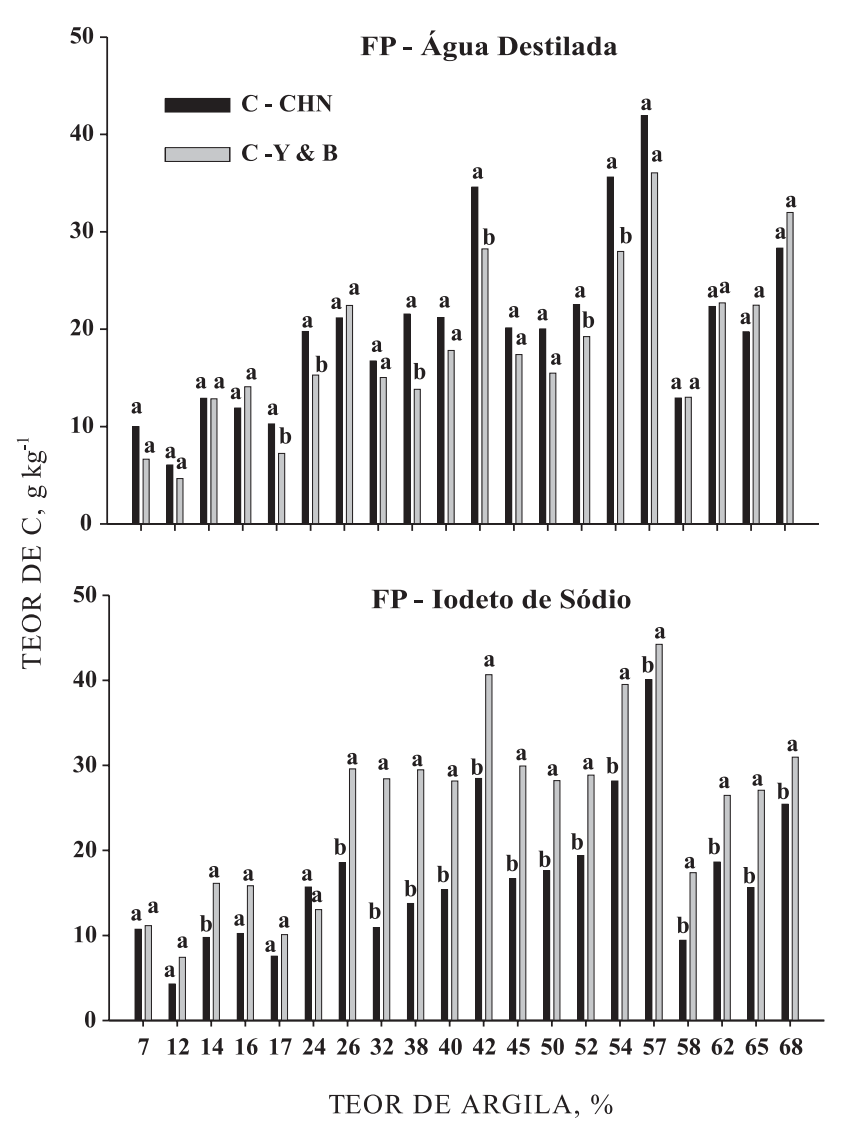

Figura 2. Conteúdo de $\mathrm{C}$ na fração pesada da matéria orgânica do solo $\left(\mathrm{g} \mathrm{kg}^{-1}\right.$ de FP) extraída com água destilada ou com iodeto de $\mathrm{Na} e$ determinado em analisador elementar (CHN) ou pelo método proposto por Yeomans \& Bremner, 1988 (Y \& B). Letras iguais para cada solo com o mesmo teor de argila não se diferem quanto aos teores de carbono, ao nível de $5 \%$ pelo teste de Tukey.

no consumo de dicromato, o que posteriormente foi quantificado como carbono. Na figura 3 é ilustrado um aumento linear nos teores equivalentes de $\mathrm{C}$ com o aumento da concentração de I-. Isso evidencia que o iodeto de $\mathrm{Na}$ interfere na leitura de $\mathrm{C}$ consumindo $\mathrm{Cr}_{2} \mathrm{O}_{7}^{-2}$ e, conseqüentemente, superestimando os valores de $\mathrm{C}$ na FP do solo, quando a FL é separada com esta solução.

As reações de oxirredução que envolvem o iodo são complexas devido aos vários níveis de oxidação possíveis $(+1,+3,+5 \mathrm{e}+7)$ (Weast, 1986). No entanto, em um exercício inicial, abaixo são apresentadas as reações mais prováveis que levam ao consumo de $\mathrm{Cr}_{2} \mathrm{O}_{7}^{-2}$ pelo NaI.

Para exemplificar o cálculo será considerado que a titulação da prova em branco $\left(0,01 \mathrm{~L}\right.$ de $\mathrm{Cr}_{2} \mathrm{O}_{7}{ }^{2-}$ consumiu $14,25 \mathrm{~mL}$ de $\mathrm{FeSO}_{4}$ 0,5 $\mathrm{mol} \mathrm{L}^{-1}(\mathrm{VB})$, e a titulação da solução após a oxidação de $\mathrm{NaI}\left(0,5 \mathrm{~mol} \mathrm{~L}^{-1}\right)$, a qual consumiu 9,49 mL de $\mathrm{FeSO}_{4}$ 0,5 mol L-1 (VA).

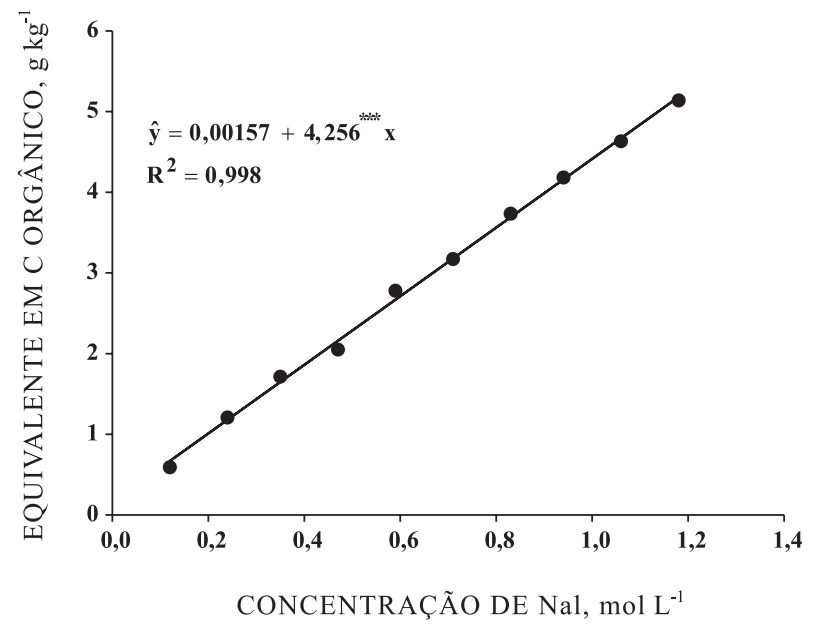

Figura 3. Equivalente em C orgânico determinados por oxidação por via úmida, em soluções isentas de $\mathrm{C}$, com concentrações crescentes de NaI.

Com a prova em branco, determinam-se exatamente quantos mols de $\mathrm{Cr}_{2} \mathrm{O}_{7}{ }^{2-}$ foram efetivamente colocados para oxidar as amostras:

mol de $\mathrm{Fe}^{2+}$ gasto na titulação dos $0,01 \mathrm{~L}$ de

$$
\begin{aligned}
& \mathrm{Cr}_{2} \mathrm{O}_{7}{ }^{-2}=\mathrm{VB} \times 0,5 \mathrm{~mol} / \mathrm{L}^{-1} \\
& \mathrm{Fe}^{2+}=14,15 \times 0,5 \mathrm{~mol} \mathrm{~L}{ }^{-1}
\end{aligned}
$$

$$
\mathrm{Fe}^{2+}=7,13 \mathrm{~mol}
$$

Na reação (2), $1 \mathrm{~mol}$ de $\mathrm{Cr}_{2} \mathrm{O}_{7}^{-2}$ é reduzido para 6 mols de $\mathrm{Fe}^{2+}$ oxidados, assim:

$$
\begin{aligned}
\text { mol de } \mathrm{Cr}_{2} \mathrm{O}_{7}-2 \text { nos } 0,01 \mathrm{~L} & =\text { mol de } \mathrm{Fe}^{2+}(1 / 6) \\
\mathrm{Cr}_{2} \mathrm{O}_{7}^{-2} \operatorname{nos} 0,01 \mathrm{~L} & =7,13(1 / 6) \\
\mathrm{Cr}_{2} \mathrm{O}_{7}-2 \operatorname{nos} 0,01 \mathrm{~L} & =1,19 \text { mols }
\end{aligned}
$$

$\mathrm{Na}$ titulação da amostra, dosa-se a quantidade de $\mathrm{Cr}_{2} \mathrm{O}_{7}^{-2}$ que não foi consumida na reação de oxidação do $\mathrm{C}$ orgânico, assim:

mol de $\mathrm{Fe}^{2+}$ gasto na titulação da amostra $=$ VA x $0,5 \mathrm{~mol} \mathrm{~L}^{-1}$

$$
\begin{gathered}
\mathrm{Fe}^{2+}=9,49 \times 0,5, \mathrm{~mol} \mathrm{~L}^{-1} \\
\mathrm{Fe}^{2+}=4,75 \mathrm{mols}
\end{gathered}
$$

$$
\begin{gathered}
\mathrm{Cr}_{2} \mathrm{O}_{7}^{-2} \text { remanescente }=\text { mol de } \mathrm{Fe}^{2+}(1 / 6) \\
\mathrm{Cr}_{2} \mathrm{O}_{7}^{-2} \text { remanescente }=4,75(1 / 6) \\
\mathrm{Cr}_{2} \mathrm{O}_{7}^{-2} \text { remanescente }=0,79 \mathrm{~mol}
\end{gathered}
$$

A diferença entre as quantidades de $\mathrm{Cr}_{2} \mathrm{O}_{7}{ }^{-2}$ adicionadas (prova em branco) e o remanescente (amostra) indica quanto de $\mathrm{Cr}_{2} \mathrm{O}_{7}^{-2}$ foi consumido na oxidação do $\mathrm{C}$ orgânico (ou iodeto):

$$
\begin{gathered}
\mathrm{Cr}_{2} \mathrm{O}_{7}^{-2} \text { consumido }=\left(\text { mol de } \mathrm{Cr}_{2} \mathrm{O}_{7}^{-2} \text { nos } 0,01 \mathrm{~L}\right)- \\
\left(\text { mol de } \mathrm{Cr}_{2} \mathrm{O}_{7}^{-2} \text { remanescente }\right) \\
\mathrm{Cr}_{2} \mathrm{O}_{7}^{-2} \text { consumido }=1,19 \text { mols }-0,79 \mathrm{~mol} \\
\mathrm{Cr}_{2} \mathrm{O}_{7}^{-2} \text { consumido }=0,40 \mathrm{~mol}
\end{gathered}
$$


Portanto, cada $0,5 \mathrm{~mol}$ de I- pode consumir 0,40 mol de $\mathrm{Cr}_{2} \mathrm{O}_{7}{ }^{-2}$. Como outros elementos também podem sofrer oxidação pelo $\mathrm{Cr}_{2} \mathrm{O}_{7}^{-2}$, como o $\mathrm{F}^{-}$, será considerado nesse exercício o consumo de $1 \mathrm{~mol}$ de $\mathrm{Cr}_{2} \mathrm{O}_{7}{ }^{-2}$ para cada mol de $\mathrm{I}^{-}$.

Com base nas reações anteriores, sugere-se a seguinte reação estequiométrica do I-:

$$
\begin{gathered}
3 \mathrm{H}_{2} \mathrm{O}+\mathrm{I}^{-} \rightarrow \mathrm{IO}_{3}^{-}+6 \mathrm{H}^{+}+6 \mathrm{e}^{-} \\
\mathrm{Cr}_{2} \mathrm{O}_{7^{-2}}+14 \mathrm{H}^{+}+6 \mathrm{e}^{-} \rightarrow 2 \mathrm{Cr}^{3+}+7 \mathrm{H}_{2} \mathrm{O} \\
\mathrm{Cr}_{2} \mathrm{O}_{7}^{-2}+8 \mathrm{H}^{+}+\mathrm{I}^{-} \rightarrow \mathrm{IO}_{3}^{-}+2 \mathrm{Cr}^{3+}+4 \mathrm{H}_{2} \mathrm{O}
\end{gathered}
$$

Assim, confirma-se a redução do cromo $\left(\mathrm{Cr}^{+6} \rightarrow\right.$ $\left.\mathrm{Cr}^{+3}\right)$ e a oxidação do iodo $\left(\mathrm{I}^{-1} \rightarrow \mathrm{I}^{+5}\right)$ e fornece-se evidência direta de que o I- proveniente do $\mathrm{NaI}$, que ficou como resíduo da separação densimétrica, é oxidado pelo dicromato, resultando em menor quantidade de dicromato remanescente do processo de oxidação e, conseqüentemente, superestimando o teor de C orgânico.

\section{CONCLUSÕES}

1. O fracionamento densimétrico da MOS que utiliza o NaI como solução de separação recupera maior quantidade da fração leve, a qual apresenta maiores teores de carbono e hidrogênio que aquela recuperada com água.

2. O resíduo de NaI presente no solo após o fracionamento densimétrico consome dicromato e leva à superestimação dos teores de $\mathrm{C}$ da fração pesada. Assim, quando do fracionamento densimétrico da MOS com o NaI, recomenda-se o uso de analisador elementar para determinar o teor de $\mathrm{C}$ orgânico das frações separadas.

3. Quando da necessidade de se usar o método da dicromatometria, o fracionamento deve ser conduzido com água destilada ou deionizada, ou outro líquido que não interfira na quantificação do $\mathrm{C}$ orgânico.

\section{AGRADECIMENTOS}

Os autores são gratos pelas valiosas sugestões de dois revisores anônimos. Em especial, gostaríamos de agradecer imensamente ao Editor Assistente, não apenas pelas críticas construtivas, mas também pela dedicada editoração.

\section{LITERATURA CITADA}

CHRISTENSEN, B.T. Physical fractionation of soil and structural and functional complexity in organic matter turnover. Eur. J. Soil Sci., 52:345-353, 2001.
CONTE, P.; SPACCINI, R.; CHIARELLA, M. \& PICCOLO, A. Chemical properties of humic substances in soils of an Italian volcanic system. Geoderma, 117:243-250, 2003.

DEFELIPO, B.V. \& RIBEIRO, A.C. Análise química do solo (metodologia). 2.ed. Viçosa, MG, Universidade Federal de Viçosa, 1997. 26p. (Boletim de Extensão, 29)

EMPRESA BRASILEIRA DE PESQUISA AGROPECUÁRIA EMBRAPA. Centro Nacional de Pesquisa de Solos. Manual de métodos de análise de solo. 2.ed. Rio de Janeiro, Ministério da Agricultura e do Abastecimento, 1997. 212p.

FREIXO, A.A.; MACHADO, P.L.O.A.; GUIMARÃES, C.M.; SILVA, C.A. \& FADIGAS, F.S. Estoques de carbono e nitrogênio e distribuição de frações orgânicas de Latossolo do cerrado sob diferentes sistemas de cultivo. R. Bras. Ci. Solo, 26:425-434, 2002b

FREIXO, A.A.; MACHADO, P.L.O.A.; SANTOS, H.P.; SILVA, C.A.; FADIGAS, F.S.; FADIGAS, F. \& SANTOS, H.P. Soil organic carbon and fractions of a Rhodic Ferralsol under the influence of tillage and crop rotation systems in southern Brazil. Soil Till. Res., 64:221-230, 2002a.

FUNDAÇÃO ARTHUR BERNARDES - FUNARBE. SAEGSistema para análise estatística. v.9.0. Viçosa, MG, 2005.

GARTEN JR., G.T.; POST, W.M.; HANSON, P.J. \& COOPER L.W. Forest soil carbon inventories and dynamics along an elevation gradient in the southern Appalachian Mountains. Biogeochemistry, 45:115-145, 1999.

KANDELER, E.; PALLI, S.; STEMMER, M. \& GERZABEK, M.H. Tillage changes microbial biomass and enzyme activities in particle-size fractions of a Haplic Chernozem. Soil Biol. Biochem., 31:1253-1264, 1999.

LIAO, J.D.; BOUTTON, T.W. \& JASTROW, J.D. Storage and dynamics of carbon and nitrogen in soil physical fractions following woody plant invasion of grassland. Soil Biol. Biochem., 38:3184-3196, 2006.

LIMA, A.M.N. Estoques de carbono e frações da matéria orgânica do solo sob povoamentos de eucalipto no Vale do Rio Doce - MG. Viçosa, MG, Universidade Federal de Viçosa, 2004. 120p. (Tese de Mestrado)

MANDO, A.; QUATTARA, B.; SÉDOGO, M.; STROOSNIJDER, L.; QUATARA, K.; BRUSSAARD, L. \& VANLAUWE, B. Long-term effect of tillage and manure application on soil organic fractions and crop performance under Sudano-Sahelian conditions. Soil Till. Res., 80:95-101, 2005.

MARRIOTT, E.E. \& WANDER, M. Qualitative and quantitative differences in particulate organic matter fractions in organic and conventional farming systems. Soil Biol. Biochem., 38:1527-1536, 2006.

MARTIN, D.; SRIVASTAVA, P.C.; GHOSH, D. \& ZECH, W. Characteristics of humic substances in cultivated and natural forest soils of Sikkim. Geoderma, 84:345-362, 1998.

MILAGRES, J.J.M. Dosagem em análises químicas de fertilidade do solo por métodos de rotina e por espectrofotometria de emissão ótica em plasma induzido. Viçosa, MG, Universidade Federal de Viçosa, 2003. 92p. (Tese de Mestrado) 
MSDS SOLUTIONS. Disponível em: http://www.msds.com/. Acesso em 20 jul. 2007.

POIRIER, N.; SOHI, S.P.; GAUNT, J.L.; MAHIEU, N.; RANDALL, E.W.; POWLSON, D.S. \& EVERSHED, R.P. The chemical composition of measurable soil organic matter pools. Organic Geochem., 36:1174-1189, 2005.

QUÉDRAOGO, E.; MANDO, A. \& STROOSNIJDER, L. Effects of tillage, organic resources and nitrogen fertilizer on soil carbon dynamics and crop nitrogen uptake in semi-arid West Africa. Soil Till. Res., 91:57-67, 2005.

ROSCOE, R. \& BUURMAN, P. Tillage effects on soil organic matter in density fractions of a Cerrado Oxisol. Soil Till. Res., 70:107-119, 2003.

SIX, J.; ELLIOTT, E.T.; PAUSTIAN, K. \& DORAN, J.W. Aggregation and soil organic matter accumulation in cultivated and native grassland soils. Soil Sci. Soc. Am. J., 62:1367-1377, 1998

SIX, J.; FELLER, C.; DENEF, K.; OGLE, S. M.; MORAES, J.C. \& ALBRECHT, A. Soil organic matter, biota and aggregation in temperate and tropical soils - Effects of no-tillage. Agronomie, 22:755-775, 2002.

SIX, J.; GUGGENBERGER, G.; PAUSTIAN, K.; HAUMAIER, L.; ELLIOTT, E.T. \& ZECH, W. Source and composition of soil organic matter fractions between and within soil aggregates. Eur. J. Soil Sci., 52:607-618, 2001.

SOHI, S.P.; MAHIEU, N.; ARAH, J.R.M.; POWLSON, D.S.; MADARI, B. \& GAUNT, J.L. A procedure for isolating soil organic matter fractions suitable for modeling. Soil Sci. Soc. Am. J., 65:1121-1128, 2001.

SOLLINS, P.; SPYCHER, G. \& GLASSMAN, C.A. Net nitrogen mineralizations from light-and heavy-fraction forest soil organic matter. Soil Biol. Biochem., 16:31-37, 1984.
STEVENSON, F.J. Humus chemistry: Genesis, composition and reactions. 2.ed. New York, Willey \& Sons, 1994. 496p.

SWANSTON, C.W.; TORN, M.S.; HANSON, P.J.; SOUTHON, J.R.; GARTEN, C.T.; HANLON, E.M. \& GANIO, L. Initial characterization of processes of soil carbon stabilization using forest stand-level radiocarbon enrichment. Geoderma, 128:52-62, 2005.

WALKLEY, A. \& BLACK I.A. An examination of Degtjareff method for determining soil organic matter and a proposed modification of the chromic acid titration method. Soil Sci., 37:29-38, 1934.

WALKLEY, A. A critical examination of a rapid and precise method for determining organic carbon in soils-effect of variations in digestion conditions and inorganic soil constituents. Soil Sci., 63:251-264, 1947.

WU, T.; SCHOENAU, J.J.; LI, F.; QIAN, P.; MALHI, S.S.; SHI, Y. \& XU, F. Influence of cultivation and fertilization on total organic carbon and carbon fractions in soils from the Loess Plateau of China. Soil Till. Res., 77:59-68, 2004.

YEOMANS, J.C. \& BREMNER, J.M. A rapid and precise method for routine determination of organic carbon in soil. Comm. Soil. Sci. Plant Anal., 13:1467-1476, 1988.

YONEBAYASHI, K. \& HATTORI, T. Chemical and biological studies on environmental humic acids: I. Composition of elemental and functional groups of humic acids. Soil Sci. Plant Nutr., 34:571-584, 1988.

WEAST, R.C. Handbook of chemistry and physics. 66.ed. Boca Raton, 1986. 2362p

WISEMAN, C.L.S. \& PÜTTMANN, W. Interactions between mineral phases in the preservation of soil organic matter. Geoderma, 134:109-118, 2006. 